\title{
Revisiting Mortality Deceleration Patterns in a Gamma-Gompertz-Makeham Framework
}

\author{
Filipe Ribeiro and Trifon I. Missov
}

\begin{abstract}
We calculate life-table aging rates (LARs) for overall mortality by estimating a gamma-Gompertz-Makeham $(\Gamma \mathrm{GM})$ model and taking advantage of LAR's parametric representation by Vaupel and Zhang [34]. For selected HMD countries, we study how the evolution of estimated LAR patterns could explain observed 1) longevity dynamics, and 2) mortality improvement or deterioration at different ages. Surprisingly, the age of mortality deceleration $x^{*}$ showed almost no correlation with a number of longevity measures apart from $e_{0}$. In addition, as mortality concentrates at older ages with time, its characteristic bell-shaped pattern becomes more pronounced. Moreover, in a $\Gamma \mathrm{GM}$ framework, we identify the impact of senescent mortality on shape of the rate of population aging. We also find evidence for a strong relationship between $x^{*}$ and the statistically significant curvilinear changes in the evolution of $e_{0}$ over time. Finally, model-based LARs appear to be consistent with point $b$ ) of the "heterogeneity hypothesis" [12]: mortality deceleration, due to selection effects, should shift to older ages as the level of total adult mortality declines.
\end{abstract}

Key words: Mortality deceleration, LAR, longevity dynamics, heterogeneity.

Filipe Ribeiro (Contact Author)

CIDEHUS.UE - University of Évora, Palácio do Vimioso - Largo do Marquês de Marialva 8, Apartado 94, 7000-809 Évora, Portugal, e-mail: fribeiro@uevora.pt

Trifon I. Missov

Max Planck Institute for Demographic Research, Konrad-Zuse-Str. 1, 18057 Rostock, Germany \& Mathematical Demography, University of Rostock, Ulmenstr. 69, 18057 Rostock, Germany, e-mail: missovedemogr.mpg.de 


\section{Introduction}

Horiuchi and Coale [11] propose a mortality measure, designated later [12, 13, 3] as the life-table aging rate (LAR), which captures the age-specific rate of mortality change for a given population. LAR, denoted by $\bar{b}(x)$ [34], is defined as

$$
\bar{b}(x)=\frac{1}{\mu(x)} \frac{d \mu(x)}{d x}=\frac{d \ln \mu(x)}{d x} .
$$

The rate of individual aging, defined as the relative derivative of the baseline hazard of death from senescent causes, is a different characteristic which is constant whenever the aging process is captured by a Gompertz curve (for further discussion on the rate of individual aging, see [20, 19]).

Gampe [6] finds partial evidence for a leveling-off of human death rates at ages 110-114. If a mortality plateau actually takes place, then it speaks in favor of a relative-risk model for adult mortality [20] with a Gompertz-Makeham baseline $\mu(x)=a e^{b x}+c[8,17]$, where $a$ measures the mortality level at the starting adult age, $b$ is the rate of individual aging, and $c$ captures the risk of dying that is not associated with the aging process. Unobserved heterogeneity (frailty) can be captured by a gamma distribution with a unit mean and $\gamma$ variance at the starting age $[31,18,20]$. This leads to the gamma-Gompertz-Makeham frailty model [31], which we will shortly address as $\Gamma \mathrm{GM}$, whose force of mortality is given by

$$
\mu(x)=\frac{a e^{b x}}{1+\frac{\gamma a}{b}\left(e^{b x}-1\right)}+c .
$$

If we estimate its parameters $a, b, c$ and $\gamma$, we can take advantage of the following formula by Vaupel and Zhang (2010) to estimate the $\Gamma G M$ model-based rate of aging for populations:

$$
\bar{b}(x)=b\left(1-\frac{c}{\mu(x)}\right)-\gamma\left(1-\frac{c}{\mu(x)}\right)(\mu(x)-c) .
$$

Horiuchi, Cheung and Robine [14] reconstructed model-based LARs by fitting a three-parameter logistic model, the Kannisto model [30], for homogeneous populations. In this study we focus on a $\Gamma G M$ heterogeneous model to reflect the perception that populations consist of individuals that share the same baseline risk of death associated with age-related deterioration of physiological functions, by senescent mortality (age-related deterioration of physiological functions), but have different (random) susceptibility to it [31]. We also incorporate a Makeham term $c$ to account for non-zero background mortality [9], i.e., mortality that cannot be directly attributed to the aging process. If $c$ is neglected, the estimates of all other $\Gamma G M$ parameters will be biased [23]. Moreover, if $c$ is left out of the model, LAR will also be distorted, and it will be impossible to capture its bell-shaped pattern [11]. The latter implies that, although age-specific mortality rates seem to follow a linear age pattern on a logarithmic scale, the rate of mortality increase is slowing down at older ages. Mortality deceleration is explained traditionally by either the heterogeneity or 
the individual-risk hypothesis [13]. The former explains observed mortality deceleration as a consequence of the existence of frailer individuals that die out at younger ages leaving a selected subpopulation of robust individuals at the older ages. This assumption implies that at the oldest ages mortality rates register a slower increase even though the hazard for individuals keeps growing exponentially [31, 33]. The individual-risk hypothesis assumes a slowdown in the rate of individual aging at older ages. In this article we assume that the heterogeneity hypothesis holds.

Ribeiro and Missov [29] show that $\Gamma G M$ model-based LAR not only fits well the observed LAR patterns after age 65, but it also captures the shift in $x^{*}$, the age of mortality deceleration, with time. The latter might suggest a relationship between the rate of increase in $e_{0}$, the life expectancy at birth, and the estimated corresponding $x^{*}$ : statistically significant changes in the slope of life-expectancy growth over time are accompanied by changes in the age of mortality deceleration. Life expectancy at birth, though, is a mortality measure that is influenced by earlylife mortality. The modal age at death $M$, on the other hand, is a characteristic of the distribution of adult deaths $[25,26,16,5,4,2,21]$ and captures mortality shifts more accurately than remaining life expectancies such as $e_{65}$ [15]. In a $\Gamma G M$ setting, getting an exact expression (see section 2.4, eq. 10) for the age of mortality deceleration $x^{*}$, i.e., the age at which LAR reaches its maximum, facilitates the study of the relationship between $x^{*}$ other longevity measures.

We fit the $\Gamma \mathrm{GM}$ model to mortality data from six selected countries to reflect different types of mortality experience: steady increase (France, Sweden and Japan), increase at a changing pace (USA) and fluctuation (Russia and Ukraine) of agespecific death rates over time. The $\Gamma \mathrm{GM}$ fits the data with high accuracy, and for the chosen countries we find evidence for point $b$ ) of the heterogeneity hypothesis ${ }^{1}$ by Horiuchi and Wilmoth [13]. Using segmented regression to identify the curvilinear structure of the three longevity measures over time, we find evidence for a plausible relationship between LAR patterns and the changes in the rate of life-expectancy increase over time, also reflected in $x^{*}$. Finally, the age of mortality deceleration $x^{*}$ seems to be strongly influenced by changes in the overall pattern of mortality.

This study not only revisits recent mortality deceleration patterns by calculating LARs for overall mortality from a $\Gamma \mathrm{GM}$ model, but also evaluates mortality dynamics by focusing on longevity development across time as a result of possible improvement or deterioration at different ages.

\section{Data and Methods}

This section presents the methodological foundations of the analysis carried out in section 3. Section 2.1 identifies the source and format of analyzed data as well as

\footnotetext{
${ }^{1}$ The hypothesis states that $a$ ) deceleration occurs for the most major causes of death (COD), being less pronounced for CODs with lower death rates and should start at earlier ages for CODs with higher death rates; and $b$ ) mortality deceleration, due to selection effects, should shift to older ages as the level of total adult mortality declines
} 
defines the time horizon of the study. Section 2.2 revisits formal definitions of remaining life expectancy $e_{x}$, the median age at death $M d$, and the modal age at death $M$. Section 2.3 presents an empirical approximation of LAR, while 2.4 introduces the LAR formula in a $\Gamma G M$ setting. Section 2.5 presents a modified version of the $\Gamma G M$ LAR by re-parameterizing the Gompertz force of mortality in terms of $M$. Finally, section 2.6 provides an overview of segmented regression, a tool that we use to single out different time intervals of linear increase in $M$ over time.

\subsection{Data}

We use overall death counts $D(x, y)$ and exposures $E(x, y)$ from the Human Mortality Database (http: / / www . mortality.org/ [10]). We focus on the period from 1970 to the last available year for each country to have, on the one hand, a common study period and, on the other hand, to avoid potential data quality problems in one or more of the selected countries (e.g., data for Ukraine [28] need to be handled carefully before 1970).

Figure 1 presents period female life expectancy at birth $e_{0}$ for the six selected countries: France, Japan, Sweden, Russia, Ukraine, and USA. Qualitatively we can identify three different patterns of $e_{0}$ over time: steady increase at almost constant pace (Sweden, Japan, France), steady increase at a changing pace (USA), and stagnation with periods of strong fluctuation (Russia, Ukraine). In the 1970s life expectancy at birth varies from 73.4 in Russia to 77.2 in Sweden. France registers the second highest value from the group with 75.8, while Japan, Ukraine, and USA share an almost identical life expectancy at birth (74.7, 74.4 and 74.7 , respectively). In the end of the observed period, Japan is leading with 86.5, while Russia and Ukraine are still on the bottom (both with 74.8 years of life expectancy at birth in the end of each correspondent series). However, USA's $e_{0}$ lags further behind the Swedish and French, and the latter surpasses the former. The selection of countries with different patterns of $e_{0}$ increase reflects our aim to understand how LAR patterns may explain (at least partially) the observed changes in the "longevity hierarchy".

For completeness of the study, we also consider cohort mortality data. However, we focus on Sweden and France, cohorts 1800-1900 only as data for these countries are characterized by both high quality and availability over a long time horizon.

\subsection{Measuring Longevity}

The age distribution of deaths is usually bimodal, representing the distinctive patterns of early and adult mortality. The distribution of adult deaths is skewed to the left and this results in $e_{0}<M d<M$. In most developed countries the gap among 
Fig. 1 Female life expectancy at birth for the studied countries from 1970 to the last available year (source: HMD 2015).

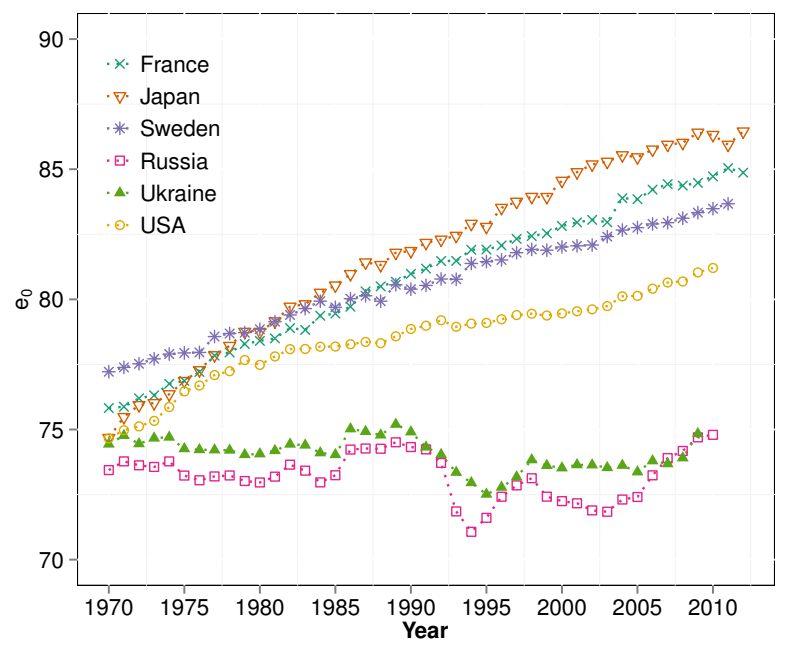

these three longevity indicators narrows down, and $M d$ is located approximately at the midpoint between $M$ and $e_{0}$ [15].

Remaining life expectancy $e_{x}$ at age $x$ can be calculated from a single-decrement life table by [27]

$$
e_{x}=\frac{T_{x}}{l_{x}},
$$

where $l_{x}$ is the proportion of individuals alive at age $x$ and $T_{x}$ denotes the personyears lived at age $x$ and above.

The median age at death $M d$ is the age by which half of the population is dead, i.e., when the survival function equals $0.5: l_{M d}=0.5$. If $l_{M d}=0.5$ in $[x, x+1)$, then the median age at death is calculated by

$$
M d=x+\frac{0.5-l_{x}}{l_{x+1}-l_{x}} .
$$

The modal age at death $M$ is the age when most adult deaths occur:

$$
M=\left\{\max _{x} d_{x}, x>5\right\},
$$

where $d_{x}$ is the life-table density function of the distribution of deaths. Kannisto [16] proposed a formula that uses life-table input to calculate the modal age at death with decimal precision. If $x$ is the age with the highest number of deaths in the life table, then 


$$
M=x+\frac{d_{x}-d_{x-1}}{\left(d_{x}-d_{x-1}\right)+\left(d_{x}-d_{x+1}\right)}
$$

\subsection{The Life-Table Aging Rate (LAR)}

If data are available for single-year age groups, the empirical rate of population aging (LAR) can be obtained by the formula [11]

$$
\bar{b}^{*}(x)=\ln (M(x))-\ln (M(x-1)),
$$

where $M(x)$ is the central death rate at age $x$. The small number of deaths at very old ages, though, leads to large stochastic variation of death rates and, consequently, a two-step procedure needs to be employed [11]:

1. applying a five-year moving average to the central death rates $M(x)$ and then calculating LAR using (8);

2. taking subsequently a triangularly weighted nine-year moving average, i.e, being the weight distributed triangularly over nine values:

$$
\bar{b}_{\text {emp }}(x)=\sum_{n=-4}^{4} \frac{5-|n|}{25} * \bar{b}^{*}(x+n)
$$

\subsection{LAR in a ГGM Framework}

Human populations are comprised of different heterogeneous subpopulations in which individuals, despite sharing the same rate of increase in the hazard of death at adult ages, are described by different levels of susceptibility [31]. Within this framework, we assume that the baseline hazard follows a Gompertz-Makeham pattern [8, 17]: $a e^{b x}+c$.

The rate of individual aging in a $\Gamma$ GM setting is captured by the relative derivative of the Gompertz part $a e^{b x}$, while the rate of aging for the entire population, i.e., LAR, equals the relative derivative of $\mu(x)$ in (2). If the former is constant over age, corresponding to a constant $b$ in (2), the latter varies age-wise and its pattern is bellshaped [11, 12, 13, 34]. LAR has been widely studied, both for human [12, 13] and non-human populations [3]. In addition, Vaupel and Zhang [34] derive in a $\Gamma$ GM setting an explicit relationship between $b$, the rate of individual mortality increase, and the LAR (see eq. 3).

The age at which the relative derivative of $\mu(x)$ reaches its maximum is the age when mortality starts decelerating. In a $\Gamma \mathrm{GM}$ framework we can derive a closedform expression for the age of mortality deceleration: 


$$
x^{*}=\frac{1}{b} \ln \left(\frac{(b+c \gamma) c}{2 a b}+\frac{\sqrt{(b+c \gamma) c \gamma[(b+c \gamma) c-4 b(a \gamma-b)]}}{2 a b \gamma}\right) .
$$

To capture accurately mortality dynamics across different periods we fit model (2). As a result, in year $y$, we capture the overall force of mortality by [32]:

$$
\mu(x, y)=\frac{a(y) e^{b(y) x}}{1+\frac{\gamma(y-x) a(y)}{b(y)}\left(e^{b(y) x}-1\right)}+c(y),
$$

where, in year $y, a(y)$ is the starting level of mortality, $b(y)$ is the rate of individual aging, $c(y)$ is the Makeham term, $\gamma(y-x)$ the variance of frailty at the initial age of analysis $x_{0}\left(x_{0}<x\right)$ among survivors from cohort $y-x$.

The fitting procedure is based on the assumption that death counts $D(x, y)$ are Poisson-distributed: $D(x, y) \sim$ Poisson $(E(x, y) \cdot \mu(x, y))$ [1], where $E(x, y)$ denotes the corresponding exposure. For each year $y$ we maximize a Poisson log-likelihood:

$$
\ln L(a(y), b(y), \gamma(y), c(y))=\sum_{x}[D(x, y) \ln \mu(x, y)-E(x, y) \mu(x, y)] .
$$

LAR follows a bell-shaped pattern at older ages [11, 12, 13] which is wellcaptured by a $\Gamma \mathrm{GM}$ model [34]. If the LAR pattern is not bell-shaped, the $\Gamma \mathrm{GM}$ approximation is not accurate (see Figure 2). As a result, it is important to identify the onset of this pattern and select it as the starting age to fit the $\Gamma \mathrm{GM}$ from. Figure 2 presents observed and $\Gamma \mathrm{GM}$ model-based (with four different starting ages) LARs for France. For both sexes, the higher the starting age of fitting the $\Gamma \mathrm{GM}$, the better the accuracy of approximating the empirical LAR. As we aim at the best fit for both sexes and across all the selected countries, we decide to start all our fitting procedures at age 65 . Note that $\Gamma \mathrm{GM}$ estimates are less accurate if a substantial proportion of the deaths in the study population occurs prior to age 65 (Russia, Ukraine and males in comparison to females, in general).

\subsection{Model-Based Modal Age at Death}

Death rates at young ages decline substantially during the first half of the $20^{\text {th }}$ century [24]. This results in a steeply increasing life expectancy at birth. From that point in time on, "the extension of length of human life in low-mortality countries is primarily due to improvements in old-age survival" [15]. Consequently, the modal age at death becomes a convenient lifespan indicator as it is not influenced by mortality at younger ages $[15,16]$.

Different stochastic models, e.g., the Gompertz, logistic and Weibull models, as well as their extensions accounting for the Makeham term [15], explain well the variation in observed adult lifespans. The Gompertz part $a e^{b x}$ can be expressed us- 
Fig. 2 Empirical and corresponding model-based LAR resulting from fitting a $\Gamma \mathrm{GM}$ with different starting ages: 50 (Model-Based 1), 55 (Model-Based 2), 60 (Model-Based 3) and 65 (Model-Based 4) for French males (a) and females (b) (Data source: HMD 2015; own estimation).

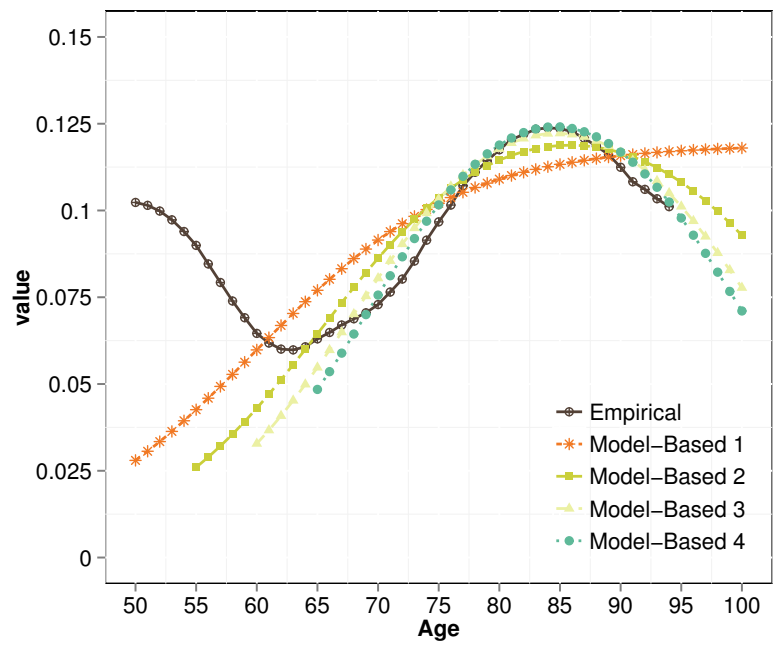

(a) Males

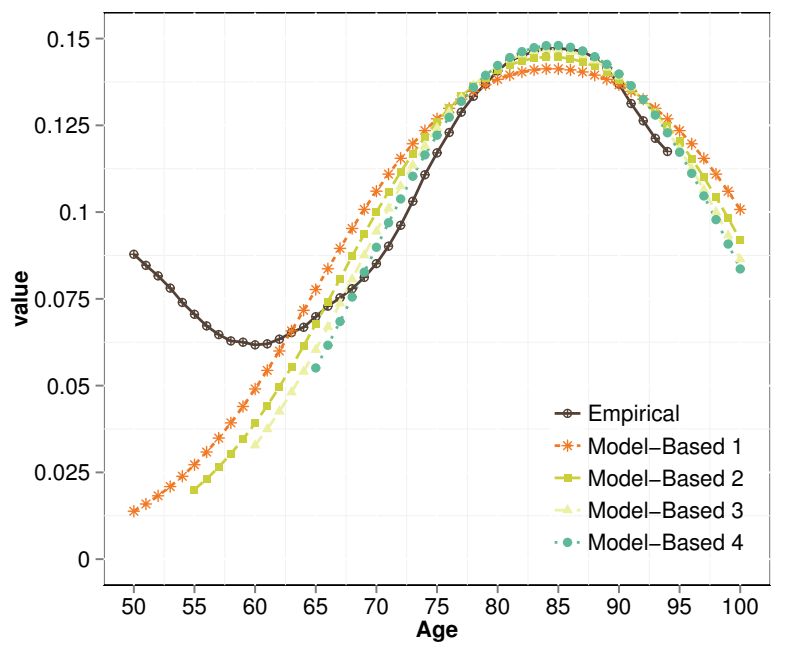

(b) Females

ing $M$ as $^{2} b e^{b(x-M)}$, and the Gompertz-Makeham force of mortality can also be re-parameterized via the Gompertz old-age mode $M: b e^{b(x-M)}+c$.

${ }^{2}$ For a detailed description please see Horiuchi et al. [15], appendix D. 
The difference between overall $M$ and the Gompertz-Makeham mode (senescent $M)$, is very small in practice. Due to the fact that at old ages the estimated level of senescent mortality is considerably higher than background mortality, the latter registers slightly higher values than the former (especially for males).

In a $\Gamma \mathrm{GM}$ framework the force of mortality can be expressed via $M$ as

$$
\mu(x)=\frac{b e^{b(x-M)}}{1+\gamma\left(e^{b(x-M)}-e^{-b M}\right)}+c,
$$

where

$$
M=\frac{1}{b} \ln \frac{b}{a} .
$$

Representing the Gompertz force of mortality in terms of $M$ rather than $a$ has also statistical advantages as the maximum-likelihood estimators of $M$ and $b$ are much less correlated than the ones of $a$ and $b$ (for broader discussion see [21]).

\subsection{Identifying the Curvilinear Structure of Longevity Measures Over Time}

To elaborate on the dynamics of the relationship between the estimated $x^{*}$ and the three longevity measures $\left(M, M d\right.$, and $\left.e_{0}\right)$ we differentiate between different period segments, i.e., we estimate a piece-wise linear model for $M$ over calendar time [22]. If we denote a break-point, i.e., a point at which the longevity measure $L M$ changes its slope, by $\psi_{i}, i=1,2, \ldots$, then

$$
L M=\alpha+\beta_{0} y_{0}+\beta_{i}\left(y_{i}-\psi_{i}\right)_{+},
$$

where $\alpha$ is the intercept, $\beta_{0}$ is the first segment slope, $\beta_{i}$ measures the difference in slopes between the first and the $i$-th segment, $\psi_{i}$ denotes the breakpoint, and $\left(y_{i}-\psi\right)_{+}=\left(y_{i}-\psi_{i}\right) \cdot I\left(y_{i}>\psi_{i}\right)$ [22]. The indicator function $I(\cdot)$ equals one when the condition in its argument is true. When the model does not detect a breakpoint, we end up with a simple linear regression, i.e., $\psi_{i}$ do not exist and $\beta_{i}$ are statistical zeroes.

\section{Results}

In this section we use the methodological framework presented in section 2 to reconstruct model-based LAR patterns and to elaborate on the link between the evolution of $x^{*}$ and the three longevity measures $\left(e_{0}, M\right.$ and $\left.M d\right)$ over time for the selected countries. Section 3.1 revisits and discusses the shape of empirical LAR patterns on human mortality surfaces. Section 3.2 studies the correlations between $e_{0}, M, M d$, 
and the age of mortality deceleration $x^{*}$. Section 3.3 focuses on $\Gamma$ GM model-based LAR patterns. Section 3.4 searches for possible relationships between the curvilinear structure of longevity measures and the corresponding old-age period mortality deceleration patterns. Finally, section 3.5 studies the connection between $x^{*}$ and $e_{0}$ on a cohort perspective.

\subsection{Revisiting Empirical LAR Patterns}

Previous studies $[11,12,13]$ acknowledge the fact that LAR follows an approximately bell-shaped pattern at ages $40+$. We take advantage of LAR's $\Gamma$ GM representation [34] (see eq. 3) to obtain a smooth parametric approximation of eq. 9. Our main goal is to reexamine empirical LAR patterns on human mortality surfaces to get better understanding about their structure and evolution over time.

According to the heterogeneity hypothesis [13], high mortality rates should yield LAR patterns with weaker curvature, and more pronounced LAR peaks should shift to later ages as mortality becomes almost exclusively concentrated at these ages (mortality compression).

Figures 3 and 4 present empirical LAR patterns at ages 30-94 for USA and Ukraine (see Appendix for LAR patterns of the other four study countries). Especially for females (USA 3(a) and Ukraine 3(b)), two LAR peaks can be identified - one at younger and another at older adult ages. Increases in LAR at young-adult ages is perhaps related to the contribution of background mortality at that stage of human life. If the first observed peak for young adults can be associated with external risks of mortality, the one at older ages reflects the increase of age-related (senescent) mortality.

In section 2.4, which focuses on selecting the best starting age, we show that it is even harder to construct accurate $\Gamma$ GM model-based LARs in the absence of a "well-defined" bell-shaped pattern, i.e., when LAR patterns are fluctuating. Consequently, the analysis of empirical LAR already anticipates possible fitting accuracy issues.

Figures 3 and 4 show that Ukrainian mortality is characterized by higher fluctuation in its LAR patterns when compared with the ones of the USA. In fact these patterns are not exclusive of Ukraine only, but are also observed in Russia (see Appendix), while the differences between males and females observed in these two figures are representative for all countries.

Figure 4 presents empirical LAR patterns for males in USA and Ukraine. In comparison with females (Figure 3), males register a much flatter and fluctuating LAR pattern in early years, while in most recent decades the peak at older ages becomes more easily distinguishable. These patterns are a consequence of higher male-associated mortality rates that, like in the female case, decrease over time as a result of mortality improvements.

Flatter LAR patterns for males in comparison with females suggests that the $\Gamma \mathrm{GM}$ model accuracy might be higher for females. As a result, the empirical LAR 
Fig. 3 Empirical LAR for females aged 30-94 in USA 1970-2010 (a) and Ukraine 1970-2009 (b) (source: HMD 2015, own calculation).

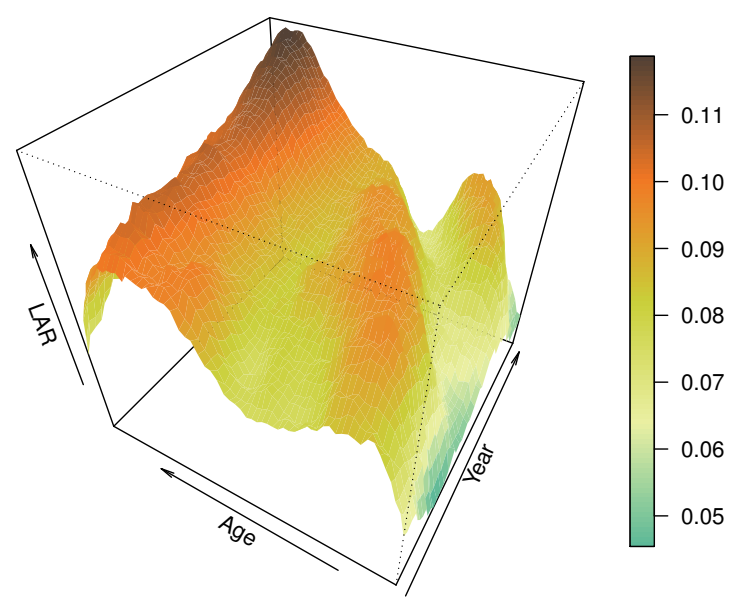

(a) USA

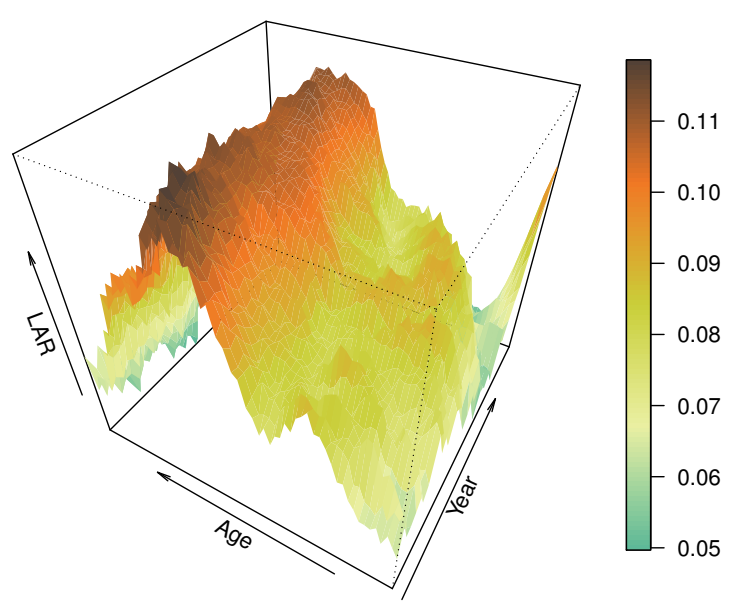

(b) Ukraine

pattern may foresee the existence of possible issues when LAR is specified in a $\Gamma G M$ framework, not only due to empirical fluctuations, but also because there is more than one peak (resulting from recent mortality improvements). One can expect, though, that in more recent years the concentration of mortality at older ages results in more accurate fits. 
Fig. 4 Empirical LAR for males aged 30-94 in USA 1970-2010 (a) and Ukraine 1970-2009 (b) (source: HMD 2015, own calculation).

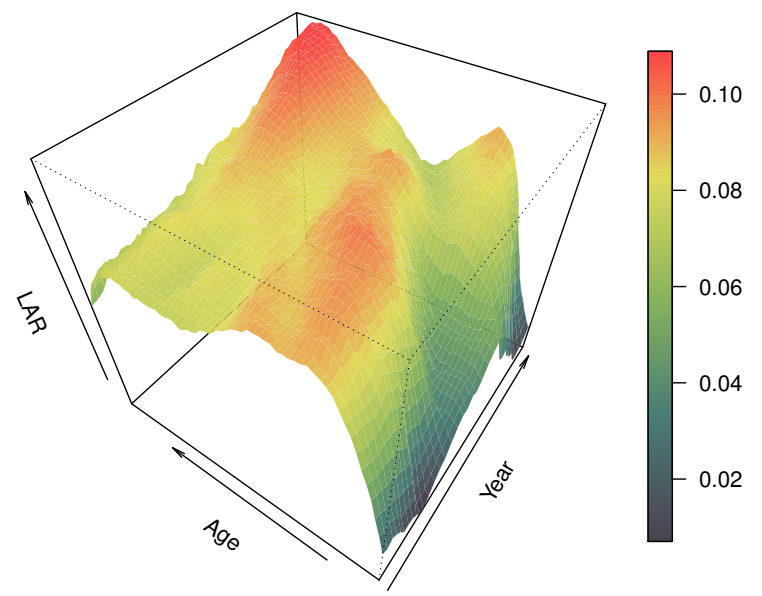

(a) USA

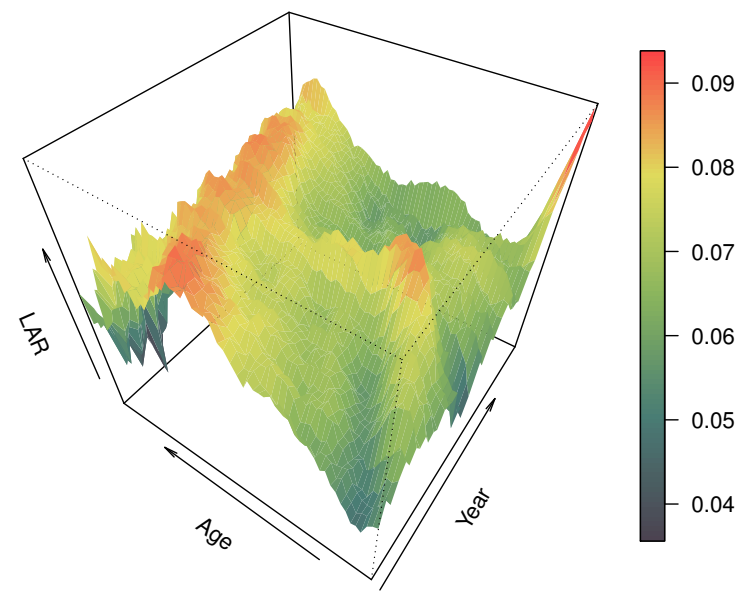

(b) Ukraine

\subsection{Correlations of Longevity Measures}

Rather than explore all possible relationships between statistically significant changes in the rate of increase across the selected longevity measures identified by the em- 
ployment of (15) and the age at which mortality starts to decelerate $x^{*}$ we perform a preliminary and exploratory evaluation of all possible relationships by calculating the correlation coefficient between each pair of measures (Table 1).

The correlation coefficient can be defined as a normalized measurement to evaluate possible linear relationships between two variables. A correlation coefficient close to 1 indicates a clear positive linear relationship between the two variables under analysis, while for values closer to -1 , it indicates a presence of a negative slope, i.e., a negative correlation. Consequently, correlation values closer to 0 indicates a weak or almost null linear relationship.

The obtained correlation coefficients presented in Table 1 indicate that in all the countries under study, exists a strictly positive relationship between $e_{0}$ and $M d$. Nevertheless, concerning the pairwise relationship $e_{0} V s M$ and $M d V s M$, except the generally high positive correlation coefficients, we find a very weak linear relationship in what concerns Russian and Ukrainian females. This weak pairwise relationship foresees the existence of an almost parallel evolution with time of the three widely used longevity measures within each relationship. Nevertheless, the small positive outcomes also suggest that in the near future longevity will possibly increase.

The statistical relationship between $e_{0} V s x^{*}, M d V s x^{*}$ and $M V s x^{*}$ in Russia and Ukraine is captured by a negative correlation, especially in the male case. As it could be seen in the previous subsection, LAR for males in Russia and Ukraine registered high fluctuation peaks at young adult ages as a result of higher mortality rates registered at those ages. This influences all three longevity measures as they decrease while $x^{*}$ increases due to more distinctive old-age LAR peaks. Despite being negative, the female correlation coefficients associated with those two pairwise comparisons are smaller. This situation can be explained by the male-female longevity gaps and sex-related pace of mortality improvements. Nevertheless, a weak positive relationship can be found between $M$ and $x^{*}$ for females, suggesting that female mortality improvements in Russia and Ukraine occur faster than the ones for males.

After the examination of possible pairwise relationships between the trends over time of "typical" longevity measures $\left(e_{0}, M d\right.$ and $\left.M\right)$ we focus on the possible relationship between the age at which LAR reaches its maximum $x^{*}$ and the three considered longevity measures. In a broader perspective, it can be seen that the relationship between $M$ and $x^{*}$ follows a weaker linear pattern. However, females living in France, Japan, Sweden and USA show high positive linear relationships for $e_{0} V s$ $x^{*}, M d V s x^{*}$ and $M V s x^{*}$. In Russia and Ukraine, independently of sex and considering the same two last pairwise relationships, the obtained results suggest a weak negative linear relationship.

The strictly positive and highly significant linear relationship between $e_{0}$ and $M d$ in Table 1 may suggest that the obtained correlation coefficients for $e_{0} V s x^{*}$ and $M d$ $V s x^{*}$ might not differ significantly or add significant information. A closer look at these two pairwise relationships reveals, though, that for $e_{0} V s x^{*}$, in presence of significant correlation values, they are slightly higher than the ones for $M d V s x^{*}$. Thus, in order to evaluate the presence of a possible connection between LAR peaks $\left(x^{*}\right)$ and longevity measures by applying (15), our choice fell on $e_{0}$. 
Table 1 Correlation coefficients between the different considered longevity measures: life expectancy at birth $e_{0}$, median $M d$ and modal $M$ age at death and the age at which mortality starts to decelerate $x^{*}$ (source: HMD 2015, own calculation).

\begin{tabular}{c|c|c|c|c|c|c|c}
\hline \hline Country & Sex & $e_{0} \boldsymbol{V} \boldsymbol{s} M d$ & $e_{0} \boldsymbol{V} \boldsymbol{s} M$ & $e_{0} \boldsymbol{V} \boldsymbol{s} x^{*}$ & $M d \boldsymbol{V} \boldsymbol{M}$ & $M d \boldsymbol{V} \boldsymbol{s} x^{*}$ & $M \boldsymbol{V} \boldsymbol{s} x^{*}$ \\
\hline \multirow{2}{*}{ France } & Male & 1.00 & 0.95 & 0.84 & 0.95 & 0.84 & 0.80 \\
& Female & 1.00 & 0.97 & 0.98 & 0.97 & 0.98 & 0.96 \\
\hline \multirow{2}{*}{ Japan } & Male & 1.00 & 0.97 & 0.65 & 0.97 & 0.65 & 0.70 \\
& Female & 1.00 & 0.98 & 0.99 & 0.98 & 0.99 & 0.96 \\
\hline \multirow{2}{*}{ Russia } & Male & 0.97 & 0.79 & -0.24 & 0.85 & -0.40 & -0.39 \\
& Female & 0.98 & 0.34 & -0.19 & 0.26 & -0.20 & 0.11 \\
\hline \multirow{2}{*}{ Sweden } & Male & 1.00 & 0.90 & 0.66 & 0.90 & 0.65 & 0.60 \\
& Female & 1.00 & 0.92 & 0.84 & 0.92 & 0.84 & 0.79 \\
\hline \multirow{2}{*}{ Ukraine } & Male & 0.99 & 0.71 & -0.61 & 0.74 & -0.65 & -0.25 \\
& Female & 0.98 & 0.05 & -0.36 & 0.06 & -0.34 & 0.11 \\
\hline \multirow{2}{*}{ USA } & Male & 1.00 & 0.96 & 0.72 & 0.96 & 0.75 & 0.68 \\
& Female & 1.00 & 0.93 & 0.97 & 0.94 & 0.95 & 0.90 \\
\hline \hline
\end{tabular}

\subsection{Model-Based Patterns}

By fitting a $\Gamma \mathrm{GM}$ model from age 65 onwards we estimate the population's rate of aging $\bar{b}(x)$ (see eq. 3 ) by country and gender. The onset of mortality deceleration observed when age-specific death rates are plotted on a logarithmic scale is reflected in the peak of the corresponding LAR pattern at age $x^{*}$. As postulated by the heterogeneity hypothesis, a fast drop-off of frailer individuals leads to a lower corresponding age of mortality deceleration. Reversely, if deaths occur later in time, $x^{*}$ increases, while the variability in the ages at death decreases. Due to this concentration of deaths in a narrow age range, the survival curve of the population becomes steeper and rectangular [35]. Like $e_{0}$ and $M d$, the age of mortality deceleration is strongly influenced by changes in the overall pattern of mortality, while $M$ is highly affected by old-age mortality.

Figure 5 presents empirical and model-based $\Gamma$ GM LAR patterns for females in the six studied countries. As already presented in Figure 2, model-based LAR captures well empirical values. However, not so well-pronounced bell-shaped patterns are associated with less accurate estimates of the $\Gamma \mathrm{GM}$ LAR. The $\Gamma \mathrm{GM}$ captures well the evolution of LAR over time (Figure 5): the flatter patterns at the beginning of the study period and the shifted patterns with stronger curvature after the 1970s. The captured shift of the age of mortality deceleration to older ages reflects point $b$ ) of the heterogeneity hypothesis: as lifespans increase, mortality deceleration occurs at older ages. Figure 5 also shows that countries with higher life expectancy register not only later mortality deceleration, but also more pronounced bell-shaped patterns (i.e., France (a), Japan (b), Sweden (c) and USA (f)). In addition, the observed (em- 
pirical) LAR values plotted after age 65 indicate that smaller population countries (such as Sweden) or countries with smaller populations after age 65 due to higher mortality rates at younger ages, present higher fluctuation patterns.

Fig. 5 Model-based LAR patterns and goodness of fit for females in France (a), Japan (b), Sweden (c), Russia (d), Ukraine (e) and USA (f) (Data source: HMD 2015; own estimation). Empirical patterns are represented by shapes - 1970: inverted triangles, 1980: diamonds, 1990: regular triangles, 2000: asterisks and 2010 (2009 for Ukraine): squares - and estimates by solid lines.

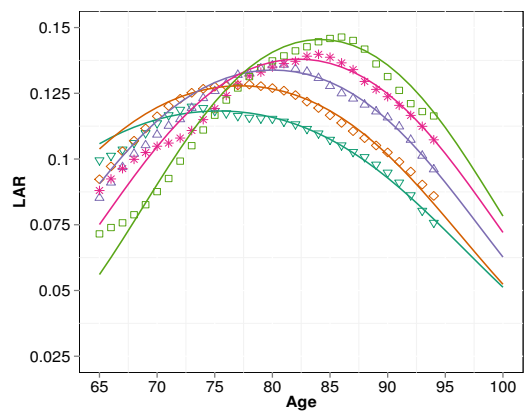

(a) France

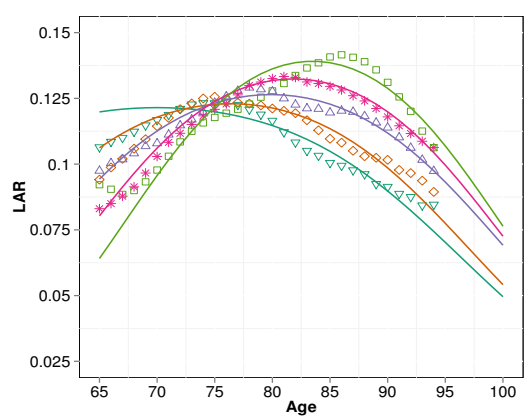

(c) Sweden

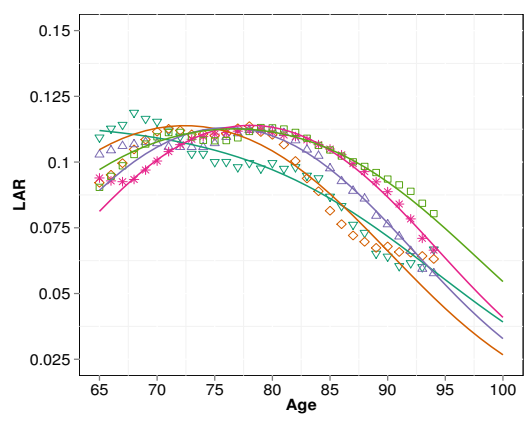

(e) Ukraine

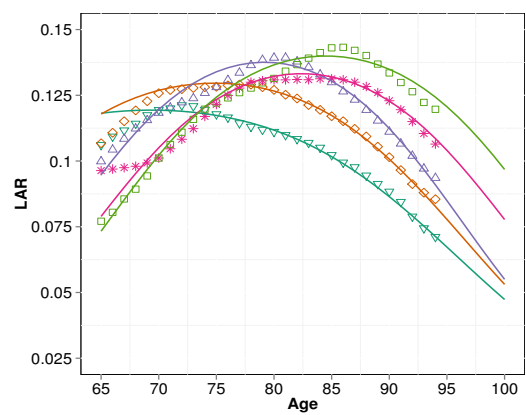

(b) Japan

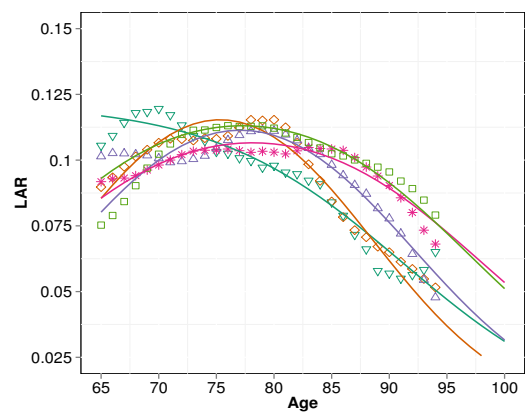

(d) Russia

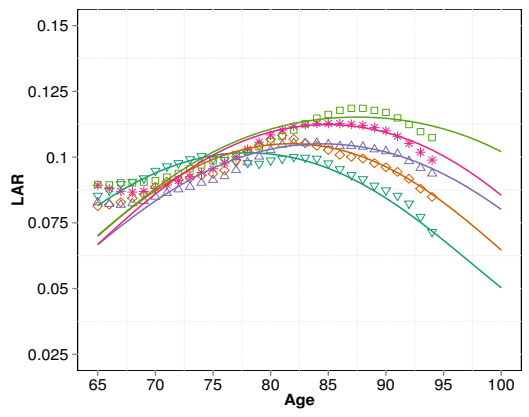

(f) USA. 
Figure 6 shows the empirical and model-based $\Gamma$ GM LAR patterns for males. In comparison to females (Figure 5), males are characterized by flatter LAR curves. Nevertheless, for almost all countries and for both sexes the age of mortality deceleration shifts to older ages with time. Deceleration starts at older ages for females, which might be connected with the longevity gap between the sexes: while female mortality is mainly concentrated at older ages, there is still "excessive" male mortality at younger ages. Estimated flatter LAR patterns, almost exclusively for males, can also be explained by higher turbulence in death rates. This is strongly pronounced in Russia and Ukraine where life expectancy has been fluctuating in the last couple of decades: Russian and Ukrainian males (6 (d) and 6 (e), respectively) are subjected to substantial turbulence in their (fairly high) mortality rates in comparison to females (Figures 5 (d) and 5 (e), respectively). As a result, LAR patterns for Russian and Ukrainian males are flat, the corresponding $\Gamma \mathrm{GM}$ approximation is quite inaccurate, and the associated age of mortality deceleration $x^{*}$ is poorly captured by equation (10). Nevertheless, $\Gamma$ GM LARs capture well the shift of mortality deceleration to older ages, as well as the observed steeper LAR curves as mortality rates decrease with time.

As described previously, flatter LAR patterns impose additional difficulties to estimate the age of mortality deceleration $x^{*}$. Figure 7 shows model-based LARs and the corresponding $x^{*}$ estimates (black circles). Although the $\Gamma G M$ model captures in general $x^{*}$ accurately, it is less problematic to identify $x^{*}$ for females than for males mainly due to the higher number of female survivors at older ages and country mortality dynamics. The estimates for Russia (7(b) and 7(d)) in Figure 7 illustrate well the estimation difficulties associated with highly fluctuating empirical LARs (see Figure 14 in the Appendix). 
Fig. 6 Model-based LAR patterns and goodness of fit for males in France (a), Japan (b), Sweden (c), Russia (d), Ukraine (e) and USA (f) (source: HMD 2015, own calculation). Empirical patterns are represented by shapes - 1970: inverted triangles, 1980: diamonds, 1990: regular triangles, 2000: asterisks and 2010 (2009 for Ukraine): squares - and estimates by solid lines.

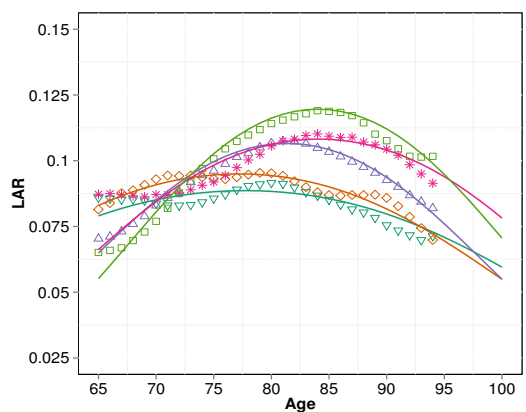

(a) France

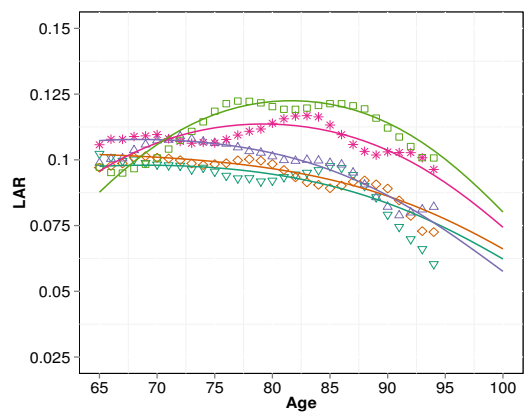

(c) Sweden

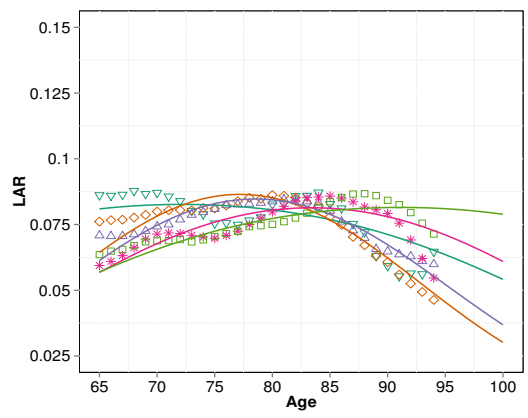

(e) Ukraine

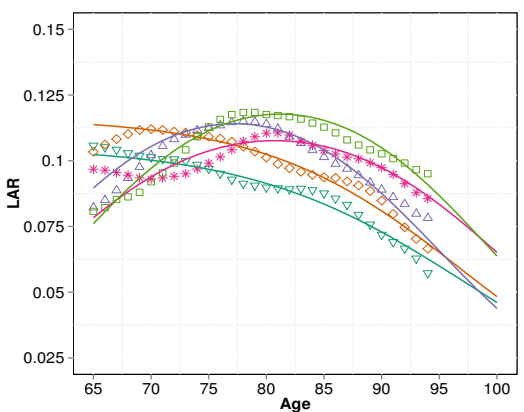

(b) Japan

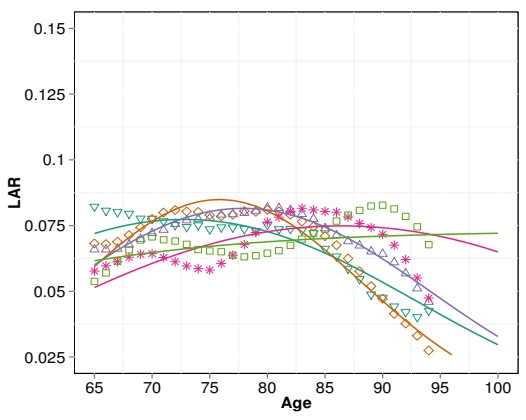

(d) Russia

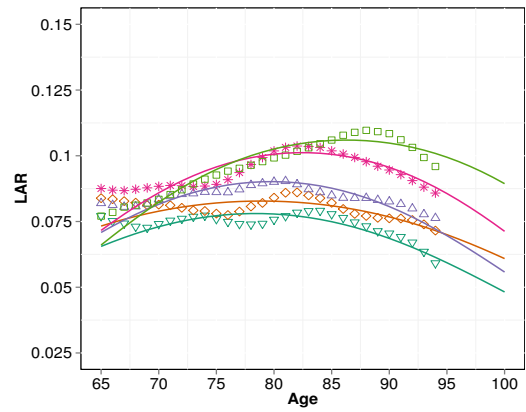

(f) USA

\subsection{LAR and Longevity for Periods}

While in section 3.1 we presented the empirical patterns of mortality deceleration, in section 3.2 we evaluate possible correlations between three of the most employed 
Fig. 7 Model-based LAR and the age of mortality deceleration $\left(x^{*}\right)$ for Females and Males in France ((a) and (b)) and Russia ((c) and (d)) (source: HMD 2015, own calculation). Image surface denotes LAR over age and year, and black circles $x^{*}$.

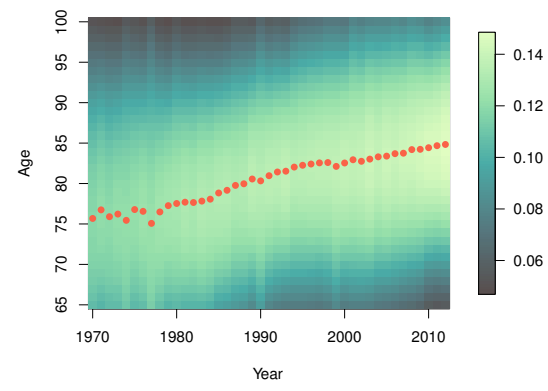

(a) France, Females

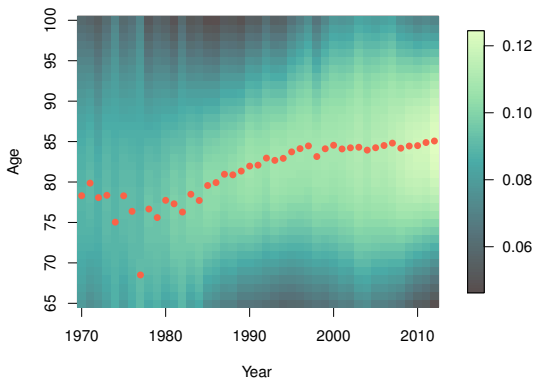

(c) France, Males

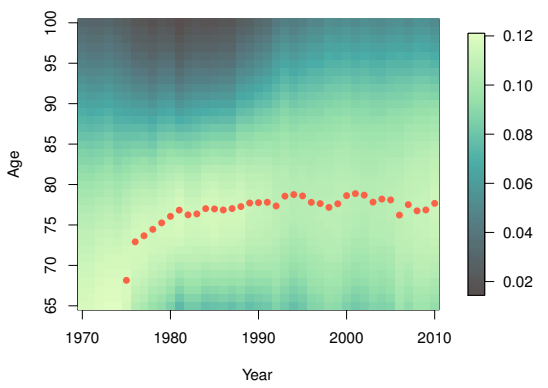

(b) Russia, Females

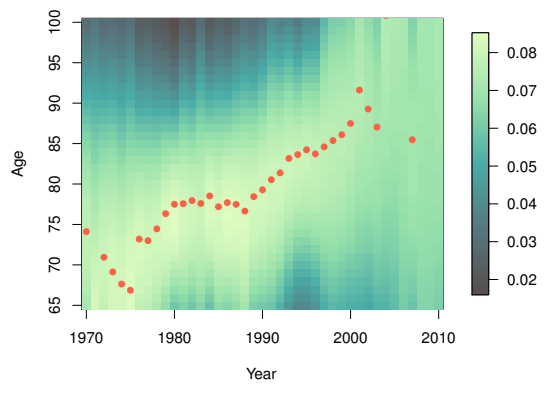

(d) Russia, Males

longevity measures and the age of mortality deceleration $x^{*}$, and in section 3.3 one can find the $\Gamma \mathrm{GM}$ parametric estimates of LAR. To provide a complementary perspective, in this section we study the evolution of longevity in the selected countries and investigate possible connections between old-age mortality deceleration patterns and life expectancy at birth $e_{0}$ (identified previously as the longevity measure with higher correlation coefficients regarding $x^{*}$ ).

In comparison to other widely-used measures of longevity like life expectancy at birth $e_{0}$, the median $M d$ and the modal age at death $M$, the age of mortality deceleration $x^{*}$ undergoes greater fluctuations: for females (Figure 8) $x^{*}$ is closer to $e_{0}$ (with the exception of USA), while in the male (Figure 9) case $x^{*}$ is closer to $M d$ for Sweden and Japan, and to $M$ in France and USA.

Figures 8 and 9 not only present the age at which mortality starts to decelerate $x^{*}$ together with three longevity measures $\left(e_{0}, M d\right.$ and $\left.M\right)$, but also the results from fitting a segmented regression to life expectancy at birth. It seems that estimated 
Fig. 8 Model-based LAR surface, segmented life expectancy at birth $e_{0}$ (dashed lines), median $M d$ (inverse triangles) and modal age at death $M$ (asterisks) and the age of mortality deceleration $x^{*}$ (circles) for Females in France (a), Japan (b), Sweden (c) and USA (d). Vertical solid lines refers to statistically significant breaks in $e_{0}$. (source: HMD 2015, own calculation)

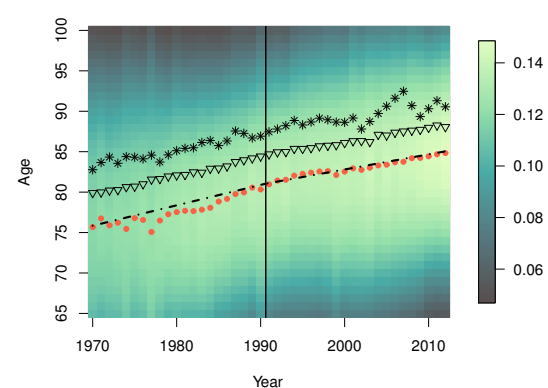

(a) France

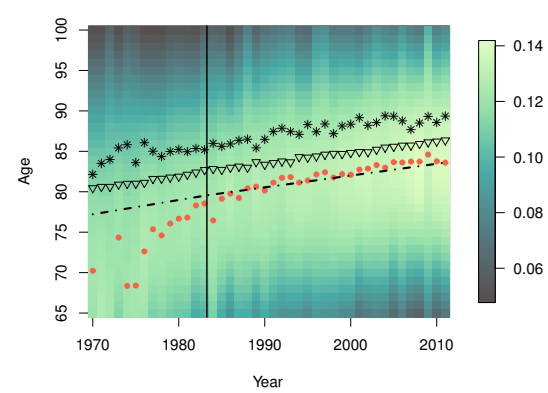

(c) Sweden

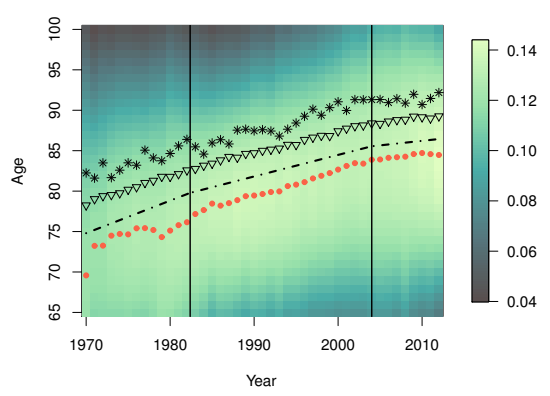

(b) Japan

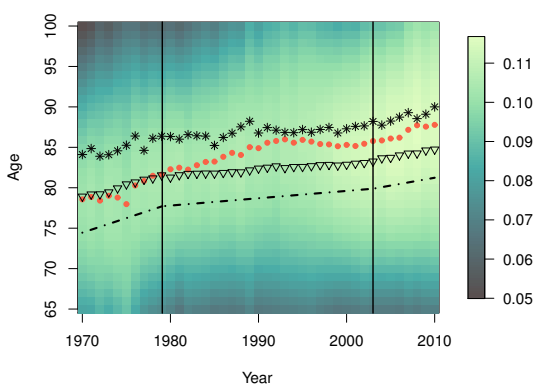

(d) USA

LAR's and the segmented dynamics of $e_{0}$ share quite high connection. Statistically significant breaks obtained for $e_{0}$ are almost always accompanied by changes in the rate of increase in $x^{*}$. Even without a strong association between significant changes in the pace of increase of $e_{0}$ and $x^{*}$, it seems that whenever a break occurs, the characteristic bell-shaped pattern curvature becomes more pronounced. 
Fig. 9 Model-based LAR surface, segmented life expectancy at birth $e_{0}$ (dashed lines), median $M d$ (inverse triangles) and modal age at death $M$ (asterisks) and the age of mortality deceleration $x^{*}$ (circles) for Males in France (a), Japan (b), Sweden (c) and USA (d). Vertical solid lines refers to statistically significant breaks in $e_{0}$. (source: HMD 2015, own calculation)

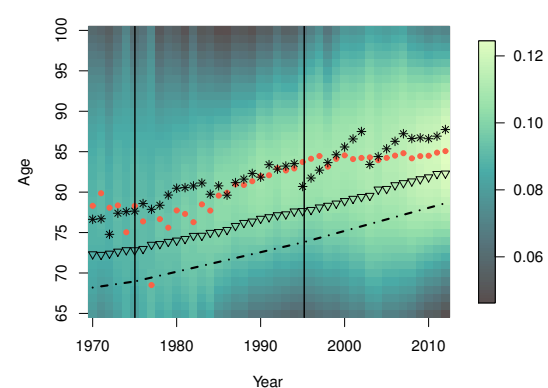

(a) France

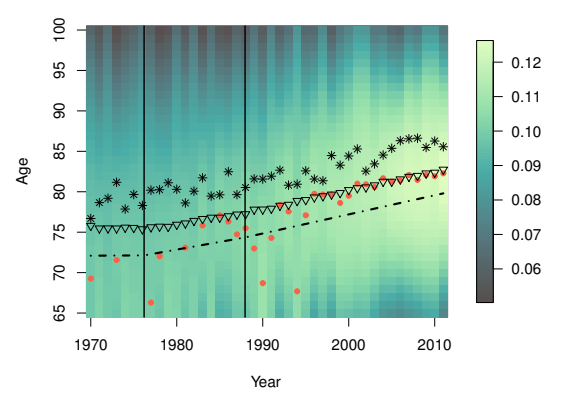

(c) Sweden

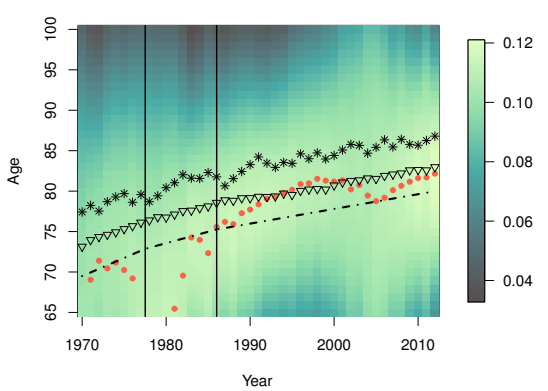

(b) Japan

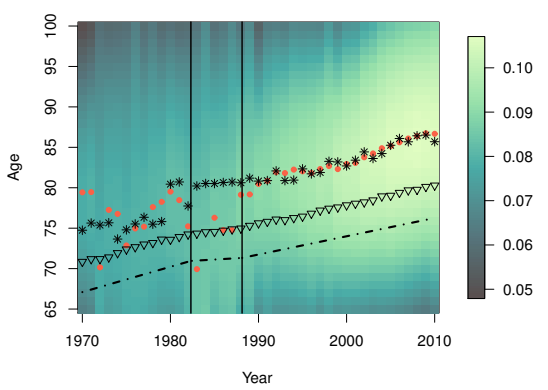

(d) USA

\subsection{LAR and Longevity for Cohorts}

For period mortality the age of mortality deceleration shifts to older ages with calendar time. This reflects the improvement of age-specific death rates and the associated selection of frail individuals at later ages in each participating cohort. For a complete overview of (empirical and model-based) LAR patterns and their evolution over time, this section focuses on cohort LAR patterns in a $\Gamma \mathrm{GM}$ setting. It aims to check whether the latter are characterized by the same evolution over time as period LAR patterns. In addition, it studies the relationship between the age of mortality deceleration for cohorts and cohort life expectancy.

To reconstruct LAR patters over a longer period we focus on single cohorts from 1800 to 1900 in France and Sweden. In comparison to empirical LAR patterns for periods, the ones for cohorts are characterized by greater fluctuation, especially for 
earlier cohorts in which the number of survivors to age 65 and above is small. This is associated with lower accuracy of the $\Gamma \mathrm{GM}$ fit (see Figure 10). LAR patterns with stronger curvature can be observed for females, but unlike the period case for earlier cohorts, the only exception being French males (see Figures 10 and 11). However, the age of mortality deceleration follows an erratic and, in the case of Sweden, even decreasing pattern (Figures 11 and 12) unlike $x^{*}$ for periods that increases steadily with calendar time. This might be due to the aforementioned greater fluctuation of cohort LAR patterns in comparison to the period ones and, on the other hand, the improvements in age-specific death rates that an aging cohort is exposed to.

Fig. 10 Empirical and $\Gamma$ GM model-based LAR patterns for France (males (a) and females (b)) and Sweden (males (c) and females (d)) for cohorts born in 1800, 1825, 1850, 1875, and 1900 (data source: HMD 2015, own calculation). Empirical patterns are represented by inverted triangles (1800), diamonds (1825), triangles (1850), asterisks (1875), and squares (1900), while $\Gamma \mathrm{GM}$ estimates are shown by solid lines.

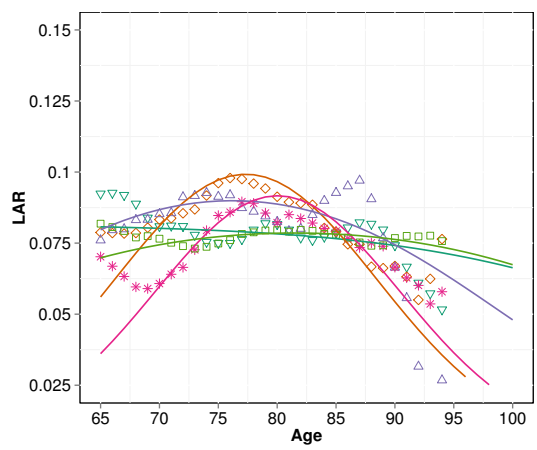

(a) France, males

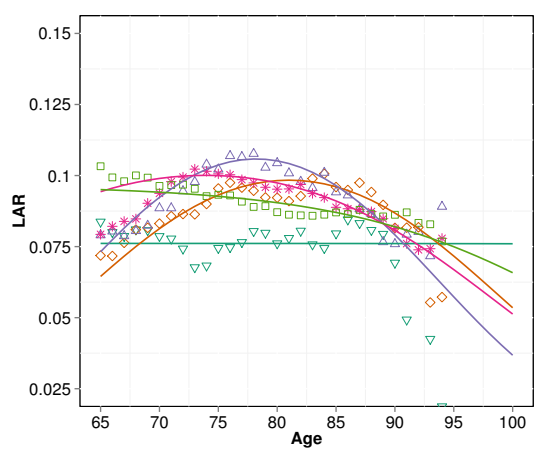

(c) Sweden, males

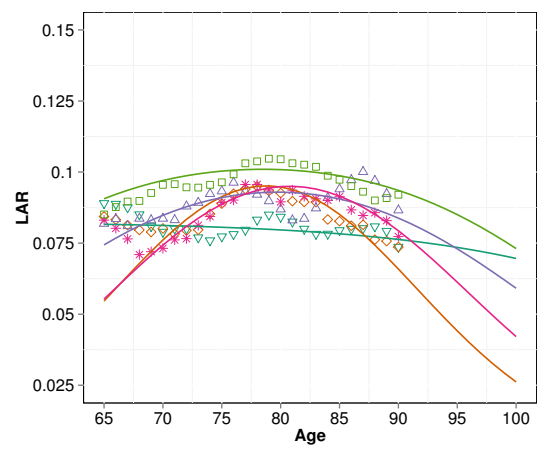

(b) France, females

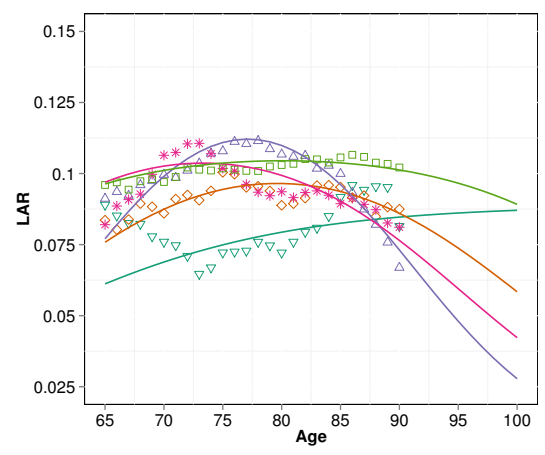

(d) Sweden, females

Figure 12 presents the age of mortality deceleration $x^{*}$ for cohorts along with cohort life expectancy at birth $e_{0}$ and the breaks identifying the segments with different 
Fig. 11 Model-based $\Gamma$ GM LAR patterns for France (males (a) and females (b)) and Sweden (males (c) and females (d)) for all cohorts born between 1800 and 1900. Lighter colors represent earlier cohorts (data source: HMD 2015, own calculation).

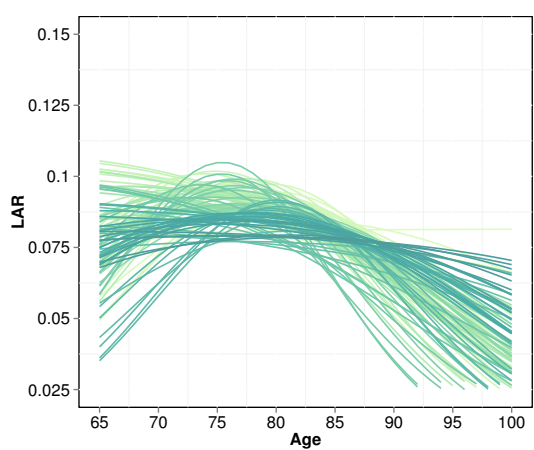

(a) France, males

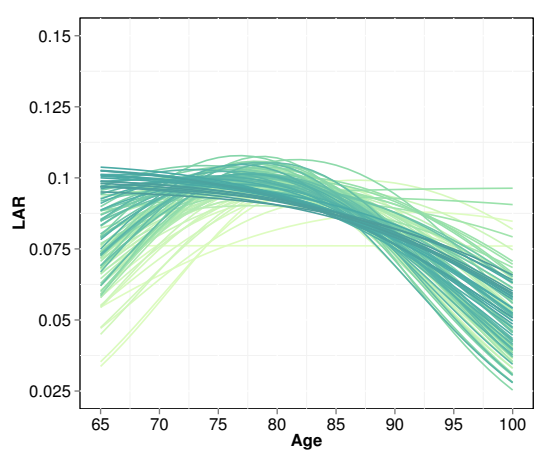

(c) Sweden, males

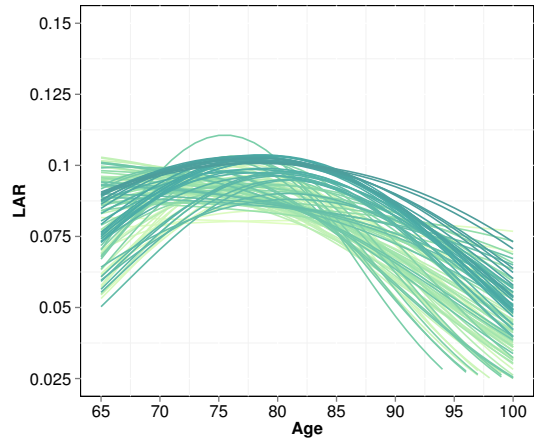

(b) France, females

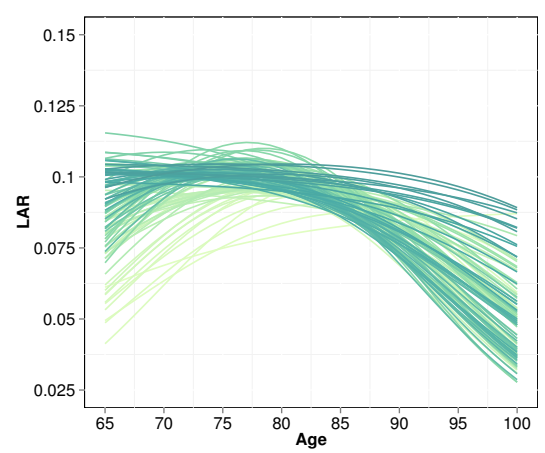

(d) Sweden, females

rates of linear increase in cohort life expectancy. The breaks for Sweden are similar between sexes, while French males and females share a common change in the rate of life expectancy increase in 1875. Like in the period case, estimated LAR pattern and the corresponding $x^{*}$ for cohorts seem to be linked to the segmented linear dynamics of $e_{0}$ : statistically significant breaks for cohort $e_{0}$ are always accompanied by changes in the rate of increase in cohort $x^{*}$, the only exception being perhaps French males (Figure 12(a)). Figure 12(d) clearly illustrates this connection: in the first segment, the slight decrease in $e_{0}$ is accompanied by decreasing $x^{*}$; in the second segment, $e_{0}$ starts to steeply increase, while $x^{*}$ seems to stay stable; in the third segment, as the pace of increase in $e_{0}$ lowers, $x^{*}$ starts to decline slowly; and in the last segment, $e_{0}$ starts to increase steeply again, while $x^{*}$ starts following the inverse pattern. Although the evolution of the two measures might be different in direction and scale, the breaks of the applied segmented regression seem to identify periods of different behavior for both measures simultaneously. 
Fig. 12 Cohort $\Gamma \mathrm{GM}$ model-based LAR surface, segmented cohort life expectancy at birth $e_{0}$ (solid line) and the cohort age of mortality deceleration $x^{*}$ (circles) for France (males (a) and females (b)) and Sweden (males (c) and females (d)). Vertical solid lines refers to statistically significant breaks in cohort $e_{0}$. (source: HMD 2015, own calculation)

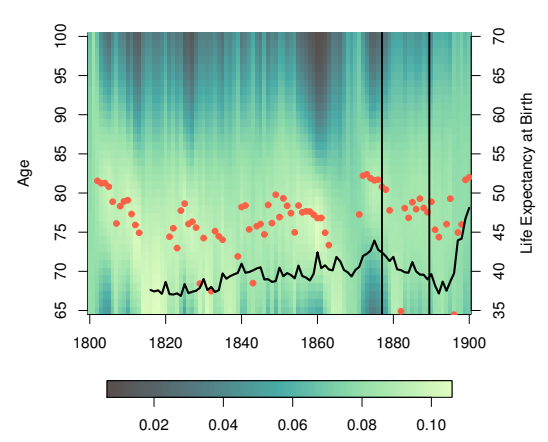

(a) France, males

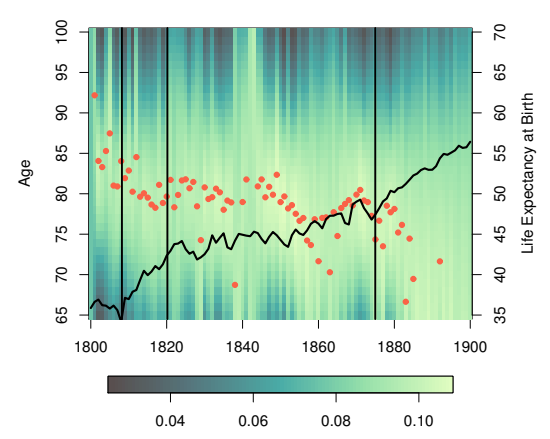

(c) Sweden, males

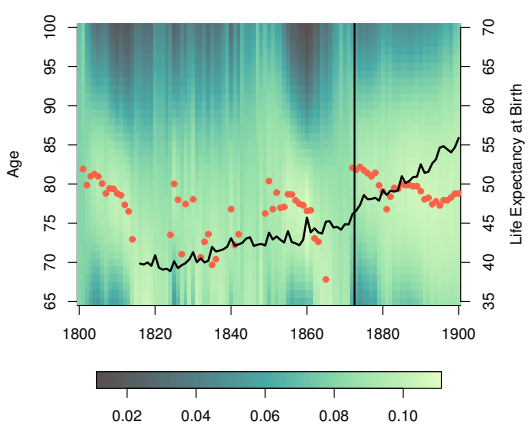

(b) France, females

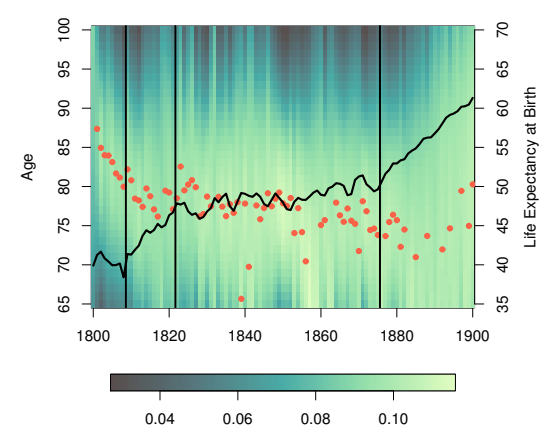

(d) Sweden, females

\section{Discussion}

A constant life-table aging rate for a population corresponds to a linear mortality increase on a logarithmic scale. LAR patterns, however, detect mortality deceleration resulting from selection of frailer individuals: in agreement with previous studies [11, 12, 13, 34], estimated and observed empirical LAR follow a bell-shaped pattern, even though the rate of individual aging, the relative derivative $b$ of the Gompertz function, is constant. An explicit relationship between the rates of individual and population aging is presented in [31,34].

We choose to work in a gamma-Gompertz-Makeham setting for two reasons: first, the $\Gamma \mathrm{GM}$ model captures with high accuracy both extrinsic mortality at younger ages (given that is convexly increasing) and mortality deceleration at older 
ages (both "tails" of the S-shaped adult-mortality curve), and, second, the $\Gamma$ GM provides adequate smooth parametric approximation to the life-table aging rate [34].

The selection of countries reflects three types of mortality experience over the last decades: steady increase, increase at a varying pace, and fluctuation of life expectancy. The inclusion of Japan in our study is important as Japan has not only registered the highest rates of mortality improvement for the last 60 years, but is also well-known for the different mortality risk factors in comparison to the ones in the European and North American countries [12]. Russia and Ukraine also deserve special attention as mortality trajectories have undergone major fluctuations for the last decades.

Stochastic variation in LAR estimates increases when the number of deaths is small [12]. The latter occurs in our study when either the country of interest has a small population size, e.g., Sweden, or a substantial proportion of deaths in a given country takes place prior to age 65 , e.g., Russia. In general, the $\Gamma \mathrm{GM}$ fits for females are better than the ones for males as at ages $65+$ the number of women exceeds the number of men. The $\Gamma \mathrm{GM}$ also captures better LAR patterns with stronger curvature.

The estimates of the $\Gamma \mathrm{GM}$ parameters $a(y), b(y), \gamma(y)$ and $c(y)$ in each year $y$ aid understanding the evolution of senescent and background mortality over calendar time. The estimated starting level of mortality $\hat{a}(y)$, i.e., mortality at age 65 , declines over time, while estimated background mortality $\hat{c}(y)$ increases with $y$. Note, however, that at ages $65+$ the share of background mortality in total mortality becomes smaller and smaller.

The increasingly higher share of senescent mortality at older ages results in a steeper bell-shaped LAR curve. However, estimating $c(y)$ is essential to capture the latter [12], and the smaller the estimates of $c(y)$, the weaker is the associated LAR's curvature (see estimates for Russia and Ukraine). Steady improvements in life expectancy result in bell-shaped patterns with stronger curvature that aid estimating LAR patterns by a $\Gamma \mathrm{GM}$ model with high accuracy (see results for France and Japan).

The estimation accuracy for the age of mortality deceleration depends on LAR's curvature: detecting $x^{*}$ for flatter the LAR patterns is more problematic. If $x^{*}$ is well approximated, the obtained results suggest a stronger correlation between this measure and both life expectancy at birth $e_{0}$ (see also [29]) and the median age at death $M d$. The high correlations in these pairs can be attributed to the fact that all three measures $\left(e_{0}, M d\right.$ and $\left.x^{*}\right)$ are sensitive to mortality at younger ages as opposed to $M$, the modal age at death. This study identifies the following relationship between statistically significant piece-wise changes in $e_{0}$ and $x^{*}$ : every change in the slope of $e_{0}$ 's linear increase is reflected in steeper LAR patterns and higher associated $x^{*}$.

$\Gamma$ GM LAR patterns for cohorts provide important insight, too. First, they confirm that mortality deceleration does not pertain (as previously hypothesized [7]) to period mortality only. Second, $\Gamma$ GM LAR patterns seem to be flatter for more recent cohorts. This can be explained, on the one hand, by the lower estimates of the Makeham term for the latest cohorts and, on the other hand, by the steady mortality improvements at ages $65+$ on a period basis (especially after 1950) that postpones 
deaths in each cohort to later ages. Finally, the evolution of cohort life expectancy seems to be related to the cohort age of mortality deceleration. Although the changes in the two mortality measures might not be the same in direction and magnitude, the statistically significant segments of curvilinear increase in $e_{0}$ correspond to segments of different functional behavior for $x^{*}$. Finding a formal relationship that links changes in $x^{*}$ to changes in life expectancy requires future study.

\section{Conclusion}

Empirical and model-based period LARs are consistent with point $b$ ) of the heterogeneity hypothesis by Horiuchi and Wilmoth [13] as mortality deceleration shifts to older ages while the level of total adult mortality declines. The age of mortality deceleration $x^{*}$, as well as life expectancy $e_{0}$ and the median age at death $M d$ seem to be strongly influenced by changes in the overall pattern of mortality, while $M$, the modal age at death, is highly affected by old-age mortality. Following the preliminary findings in [29], this study finds a relationship between changes in the rate of life-expectancy increase with time and the corresponding LAR patterns: each breakpoint in the curvilinear evolution of $e_{0}$ results in steeper LAR patterns. A similar pattern is observed for cohort LAR patterns, too. However, the evolution of the age of mortality deceleration for cohorts is more erratic, and, in the case of Sweden, $x^{*}$ is even decreasing. Nevertheless mortality deceleration is clearly seen for both periods and cohorts, and the link between its onset and different longevity measures is still to be formalized. 


\section{Appendix}

Fig. 13 Empirical LAR for females aged 30-94 in Japan (a) and France (b) 1970-2012, Sweden (c) 1970-2011 and Russia (d) 1970-2010 (source: HMD 2015, own calculation).

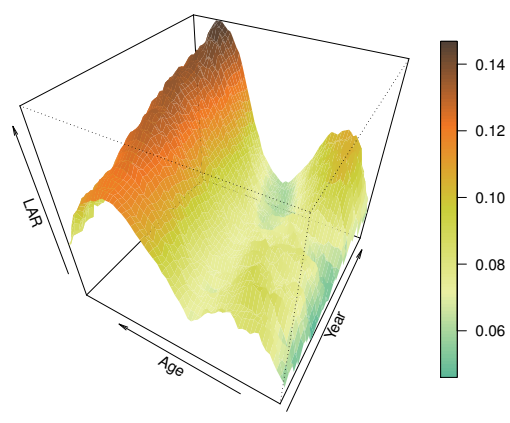

(a) France

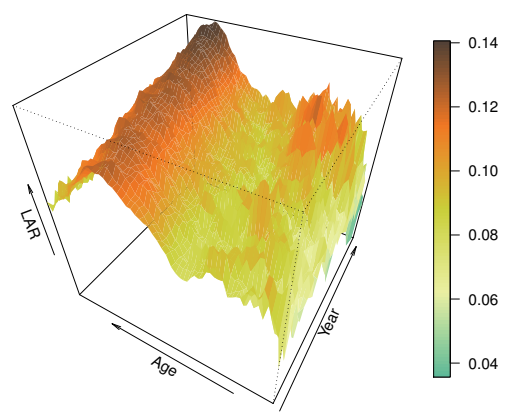

(c) Sweden

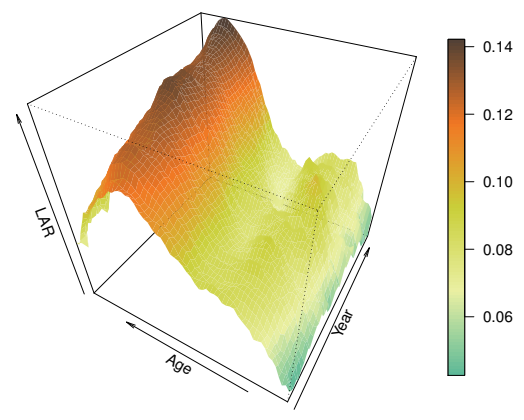

(b) Japan

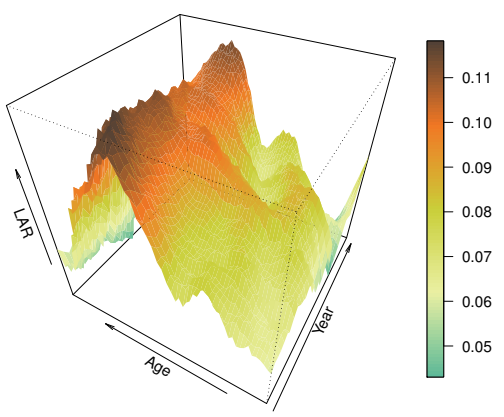

(d) Russia 
Fig. 14 Empirical LAR for males aged 30-94 in Japan (a) and France (b) 1970-2012, Sweden (c) 1970-2011 and Russia (d) 1970-2010 (source: HMD 2015, own calculation).

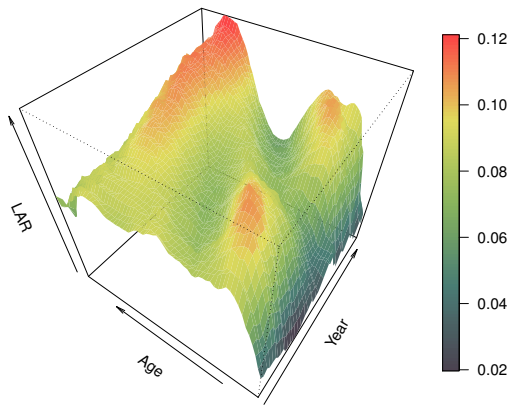

(a) France

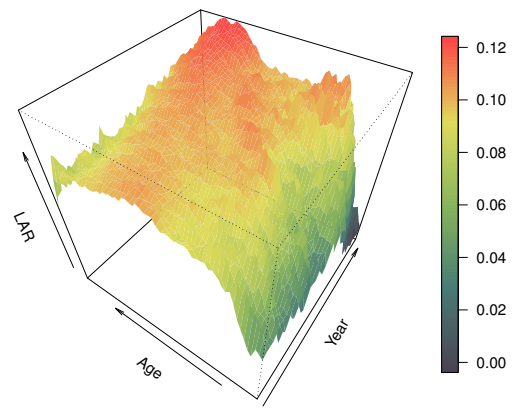

(c) Sweden

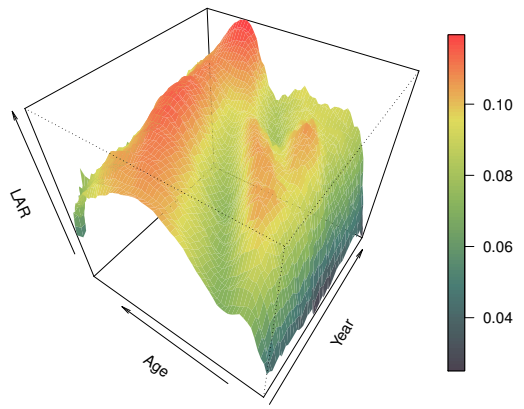

(b) Japan

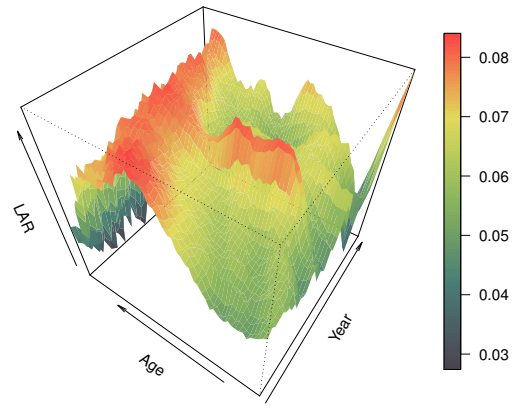

(d) Russia 
Fig. 15 Model-based LAR and the age of mortality deceleration ( $\left.x^{*}\right)$ for females in Japan (a), Sweden (b), Ukraine ((c) and USA (d) (source: HMD 2015, own calculation). Image surface denotes LAR over age and year, and black circles $x^{*}$.

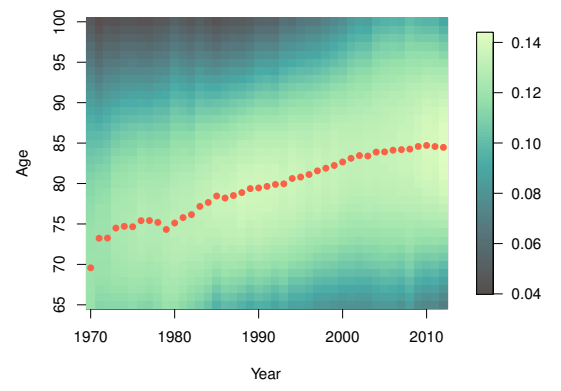

(a) Japan

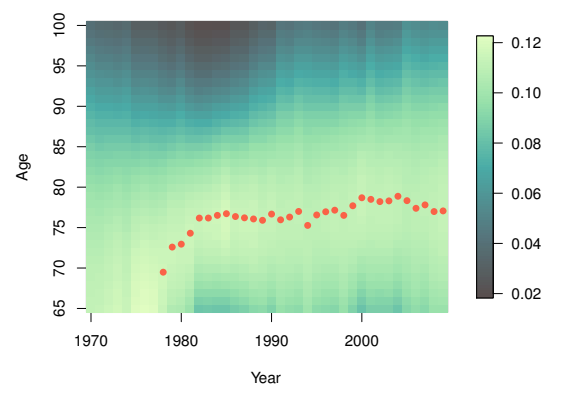

(c) Ukraine

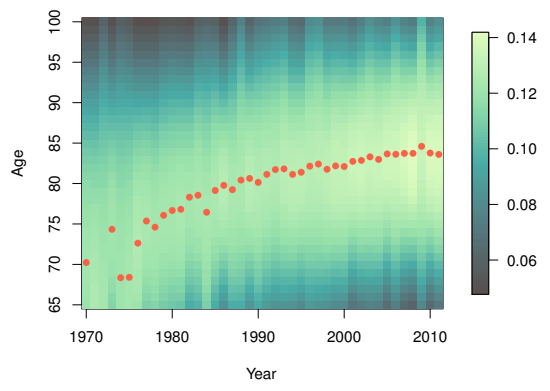

(b) Sweden

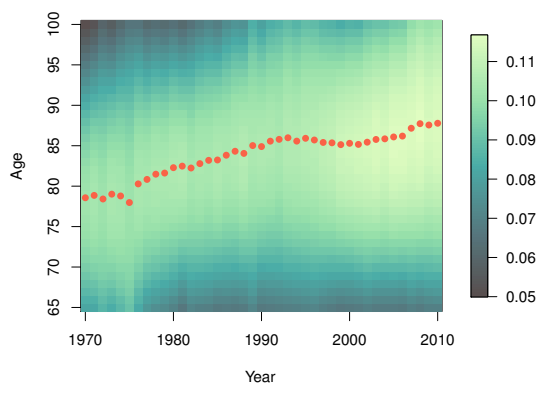

(d) USA 
Fig. 16 Model-based LAR and the age of mortality deceleration $\left(x^{*}\right)$ for males in Japan (a), Sweden (b), Ukraine ((c) and USA (d) (source: HMD 2015, own calculation). Image surface denotes LAR over age and year, and black circles $x^{*}$.

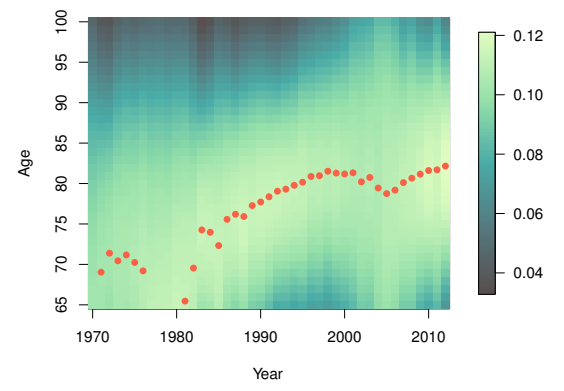

(a) Japan

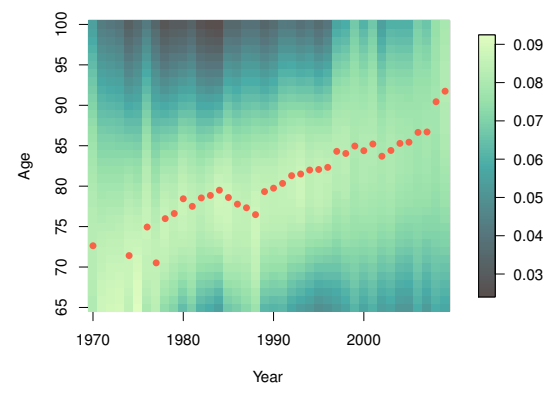

(c) Ukraine

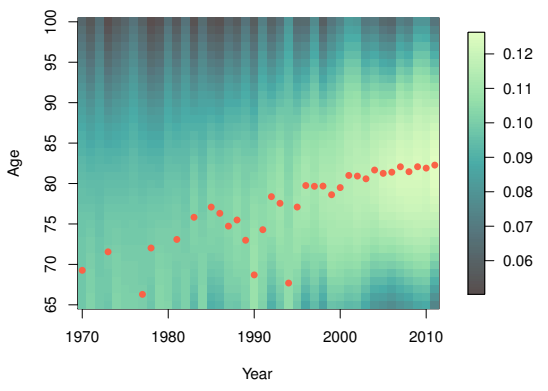

(b) Sweden

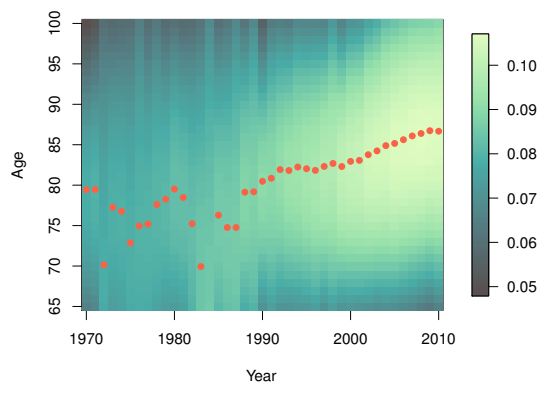

(d) USA 


\section{References}

1. Brillinger, D.R. (1986). The natural variability of vital rates and associated statistics. Biometrics 42, 693-734.

2. Canudas-Romo, V. (2008). The modal age at death and the shifting mortality hypothesis. Demographic Research 19(30): 659-686.

3. Carey, J.R., Liedo, P. (1995). Sex-specific life table aging rates in large medfly cohorts. Experimental Gerontology 30, 315-325.

4. Cheung, S.L.K., Robine, J.M. (2007). Increase in common longevity and the compression of mortality: The case of Japan. Population Studies 19(30), 85-97.

5. Cheung, S.L.K., Robine, J.-M., Tu, E.J.C., Caselli, G. (2005). Three dimensions of the survival curve: Horizontalization, verticalization, and longevity extension. Demography 42(2), 243-258.

6. Gampe, J. (2010). Human mortality beyond age 110. In: H. Maier, J.G.B. Jeune, J.M. Robine, and J.W. Vaupel (eds), Supercentenarians, Heidelberg, Springer, 219-230.

7. Gavrilov, L.A., Gavrilova, N.S. (2011). Mortality measurement at advanced ages: A study of the social security administration death master file. North American Actuarial Journal 15(3), $442-447$.

8. Gompertz, B. (1825). On the nature of the function expressive of the law of human mortality, and on a new mode of determining the value of life contingencies. Philosophical Transactions of the Royal Society of London 115, 513-585.

9. Golubev, A. (2004). Does Makeham make sense? Biogerontology 5, 159-167

10. HMD (2015). The Human Mortality Database. http: //www.mortality.org/ . Accessed on January 23, 2015.

11. Horiuchi, S., Coale, A.J. (1990). Age patterns of mortality for older women: An analysis using the age-specific rate of mortality change with age. Mathematical Population Studies 2(4), 245-267.

12. Horiuchi, S., Wilmoth, J.R. (1997). Age patterns of the life table aging rate for major causes of death in Japan, 1951-1990. Journal of Gerontology: Biological Sciences 52A(1), B67-B77.

13. Horiuchi, S., Wilmoth, J.R. (1998). Deceleration in the age pattern of mortality at older ages. Demography 35(4), 391-412.

14. Horiuchi, S., Cheung, S.L.K., Robine, J.M. (2012). Cause-of-death decomposition of old-age mortality compression. In: 2012 annual meeting of the Population Association of America (PAA), San Francisco, USA.

15. Horiuchi, S., Ouellette, N., Cheung, S.L.K., Robine, J.M. (2013). Modal age at death: Lifespan indicator in the era of longevity extension. Vienna Yearbook of Population Research 11, 37-69.

16. Kannisto, V. (2001). Mode and dispersion of length of life. Population: An English Selection 13, 159-171.

17. Makeham, W.M. (1860). On the law of mortality. Journal of the Institute of Actuaries 13, 283-287.

18. Missov, T.I., Finkelstein, M. (2011). Admissible mixing distributions for a general class of mixture survival models with known asymptotics. Theoretical Population Biology 80(1): 6470 .

19. Missov, T.I., Ribeiro, F. (2015). Do individuals age at the same rate? Findings from cause-ofdeath data. Demography forthcoming.

20. Missov, T.I., Vaupel, J.W. (2015). Mortality implications of mortality plateaus. SIAM Review 57(1), 61-70.

21. Missov, T.I., Lenart, A., Nemeth, L., Canudas-Romo, V., Vaupel, J.W. (2015). The Gompertz force of mortality in terms of the modal age at death. Demographic Research 32(36), 10311048.

22. Muggeo, V. (2003). Estimating regression models with unknown break-points. Statistics in Medicine 22, 3055-3071. 
23. Nemeth, L., Missov, T.I. (2014). How wrong could parameter estimates be? Statistical consequences of fitting the wrong model to human mortality data. In: 2014 European Population Conference, Budapest, Hungary.

24. Oeppen, J., Vaupel, J.W. (2002). Broken limits to life expectancy. Science 296, 1029-1031.

25. Pollard, J.H. (1998). An old tool-modern applications. Research Paper Series No. 001/98. School of Economic and Financial Studies, Macquarie University Sydney NSW Australia.

26. Pollard, J.H. (1998). Keeping abreast of mortality change. Research Paper Series No. 002/98. School of Economic and Financial Studies, Macquarie University Sydney NSW Australia.

27. Preston, S., Heuveline, P., Guillot, M. (2001). Demography: Measuring and Modeling Population Processes. Willey-Blackwell.

28. Pyrozhkov, S., Foygt, N., Jdanov, D. (2011). About mortality data for Ukraine. Human Mortality Database Background and Documentation.

29. Ribeiro, F., Missov, T.I. (2014). Mortality inferences from estimated life-table aging rates in a gamma-Gompertz-Makeham framework. In: 2014 Annual Meeting of the Population Association of America (PAA), Boston, USA.

30. Thatcher, A.R., Kannisto, V., Vaupel, J.W. (1998). The Force of Mortality at Ages 80 to 120. Odense: Odense University Press, Monographs on Population Aging 5.

31. Vaupel, J.W., Manton, K., Stallard, E. (1979). The impact of heterogeneity in individual frailty on the dynamics of mortality. Demography 16, 855-860.

32. Vaupel, J.W., Missov, T.I. (2014). Unobserved heterogeneity: A review of formal relationships. Demographic Research 31(22), 659-686.

33. Vaupel, J.W., Yashin, A.I. (1985). Heterogeneity's ruses: Some surprising effects of selection on population dynamics. The American Statistician 39, 176-185.

34. Vaupel, J.W., Zhang, Z. (2010). Attrition in heterogeneous cohorts. Demographic Research, 23(26), 737-748.

35. Wilmoth, J.R., Horiuchi, S. (1999). Rectangularization revisited: Variability of age at death within human populations. Demography 4(36): 475-495. 